\title{
Spatial memory impairments in amnestic mild cognitive impairment in a virtual radial arm maze
}

\author{
This article was published in the following Dove Press journal: \\ Neuropsychiatric Disease and Treatment \\ 17 April 2014 \\ Number of times this article has been viewed
}

\author{
Jun-Young Lee ${ }^{1,2}$ \\ Sooyeon Kho',2 \\ Hye Bin Yoo ${ }^{1,2}$ \\ Soowon Park ${ }^{1,2}$ \\ Jung-Seok Choi ${ }^{1,2}$ \\ Jun Soo Kwon ${ }^{3}$ \\ Kyung Ryeol Cha ${ }^{4}$ \\ Hee-Yeon Jung ${ }^{1,2}$ \\ 'Department of Psychiatry, \\ Seoul National University, ${ }^{2}$ Seoul \\ Metropolitan Government - Seoul \\ National University Boramae Medical \\ Center, ${ }^{3}$ Department of Psychiatry, \\ Seoul National University College \\ of Medicine, Seoul, South Korea; \\ ${ }^{4}$ Department of Psychiatry, Osan \\ Mental Hospital, Gyeonggi, South \\ Korea
}

Correspondence: Kyung Ryeol Cha Department of Psychiatry, Osan Mental Hospital, 543-I, Gwol-dong,

Osan-si, Gyeonggi-do, South Korea

Tel +82 3I 3702468

Email newcha@gmail.com

Hee-Yeon Jung

Department of Psychiatry,

Seoul National University and Seoul

Metropolitan Government - Seoul

National University Boramae Medical

Center, Sindaebang 2-dong, Dongjak-gu,

Seoul 156-707, South Korea

Tel +82 22870246 I

Email hyjung@snu.ac.kr
Objective: This study aims to apply the virtual radial arm maze (VRAM) task to find spatial working memory and reference memory impairments in patients of amnestic mild cognitive impairment (aMCI) and Alzheimer's disease (AD). Spatial memory functions between aMCI converters and nonconverters are also compared using VRAM results.

Methods: We assessed the spatial memory in 20 normal controls, 20 aMCI, and 20 mild AD subjects using VRAM. The Mini-Mental State Examination, Clinical Dementia Rating scale, and other neuropsychological tests were given to the subjects in conjunction with the VRAM test. Scores in working memory errors and reference memory errors were compared among the three groups using repeated measures analysis of variance. In addition, aMCI patients were followed-up after 5 years and surveyed for $\mathrm{AD}$ conversion rate.

Results: In $\mathrm{AD}$ patients, both spatial working and reference memory were impaired. However, in aMCI subjects, only spatial reference memory was impaired. Significant spatial reference memory impairment was found in the aMCI converter group when compared to the nonconverter group.

Conclusion: Spatial working memory is less impaired in aMCI while reference memory is similarly damaged in AD. In aMCI patients, more severe spatial reference memory deficit is a neuropsychological marker for AD conversion. VRAM may be well utilized in humans to assess spatial memory in normal aging, in aMCI, and in AD.

Keywords: spatial behavior, Alzheimer's disease, user computer interface, cognition

\section{Introduction}

In most cases, Alzheimer's disease (AD) is preceded by a prodromal stage and amnestic mild cognitive impairment (aMCI) in which an individual's memory is impaired to a greater degree than expected given the individual's age, sex, and educational background, while the individual's ability to perform the activities of daily living is preserved and the criteria for dementia are not met. A previous study reported that the conversion rate of aMCI to $\mathrm{AD}$ is $10 \%-15 \%$ annually, compared with $1 \%-2 \%$ among normal elderly individuals. ${ }^{1}$

Structural and functional abnormalities in the hippocampus have been documented in patients with aMCI and AD. ${ }^{2,3}$ Atrophy of hippocampal formation is a strong risk factor of AD progress. ${ }^{4}$ As the hippocampus plays a crucial role in spatial memory and navigation, ${ }^{5}$ it is generally accepted that patients with aMCI or AD show impairments in spatial orientation, ${ }^{6}$ navigation, ${ }^{7}$ and visuospatial short-term memory. ${ }^{8}$ Such deficits may lead to failure in navigating and remembering places. 
However, it is difficult to measure spatial memory deficits in real situations with a classic neuropsychological battery. Several studies utilized virtual reality systems to assess cognitive impairments related to degenerative changes to improve ecological validity. ${ }^{7,9-12}$ Previous studies reported that virtual navigation performance was impaired in aMCI and AD and that virtual environments provide valid assessments of navigation skills that are comparable to those required in real world navigation. ${ }^{9,13}$ Moreover, examination of spatial memory can be effectively performed using virtual systems, resulting in enhanced diagnostic power compared with classic neuropsychological testing. ${ }^{14}$

The virtual radial arm maze (VRAM), virtual Morris water maze, and virtual route learning are tools commonly used in assessments of spatial memory. ${ }^{15-17}$ The VRAM, a human analogue of the radial arm maze (RAM) test used extensively to test spatial memory in small animals such as rodents, ${ }^{18,19}$ has two merits to examine spatial working memory and reference memory simultaneously when compared to the virtual Morris water maze and virtual route learning. The current VRAM design uses a computer-human interface, and human subjects use monitors and joysticks to control their movements inside the virtual reality in which the classic design of RAM is implemented. The VRAM has been successfully used in human studies on sex differences in these two types of spatial memory, ${ }^{20}$ on working memory load in elderly patients, ${ }^{21}$ and on hippocampal activity. ${ }^{22}$

The hippocampus and prefrontal cortex of rodents and humans are involved in spatial navigation. ${ }^{22,23}$ Evidence from experiments with rodents indicates that memory functions involve information processing in the hippocampus and prefrontal cortex. ${ }^{24,25}$ However, spatial reference and working memory systems use different neuronal networks; ${ }^{26}$ the prefrontal cortex is involved in working memory, whereas the hippocampal region is involved in reference memory. ${ }^{12}$ A study in rodents also reported that deficits in the two types of spatial function have different patterns; reference memory deficits are common as a function of aging, but working memory deficits are not. ${ }^{27}$ As in rodents, spatial reference memory in humans is mediated by the hippocampus, ${ }^{28}$ whereas spatial working memory is more closely related to the frontal cortex. ${ }^{29,30}$

This study aimed to identify spatial memory impairment in patients with aMCI and AD using the VRAM and to determine whether the VRAM can differentiate between the two types of memory deficits. Beginning with the onset of aMCI, patients with AD typically show early changes in the hippocampus. ${ }^{31}$ Thus, we expected that both aMCI and AD patients would have reference memory deficits attributable to hippocampal atrophy. ${ }^{32} \mathrm{AD}$ pathology is also associated with spatial working memory problems as measured by the Spatial Span Backward test. ${ }^{31}$ This may be related to the fact that prefrontal cortical atrophy is more prominent in $\mathrm{AD}$ than in $\mathrm{aMCI} .{ }^{33}$ Therefore, we expected that spatial reference memory deficits would appear in both aMCI and AD patients and working memory loss would be found only in patients with AD. We also followed aMCI participants for 5 years to find a virtual navigation predictor of progression from aMCI to AD. ${ }^{1}$

\section{Methods}

Subjects

Sixty older adults were recruited from the local community. The Institutional Review Board at Boramae Hospital approved the study protocol. All procedures in this study were in accordance with the ethical standards of the responsible committee on human experimentation and with the Helsinki Declaration of 1975, as revised in 1983. Eligibility criteria for participation were 1) age 60 years or older and 2) no known history of head trauma, brain tumor, stroke, mental retardation, or any severe medical, neurological, or psychiatric illness affecting cognitive functioning other than AD. Patients with mild depression were included only if their scores on the short form of the Geriatric Depression Scale were lower than $10 .{ }^{34}$ All participants were assigned to one of three groups: normal control (NC) $(n=20)$, aMCI $(n=20)$, or mild AD $(n=20)$. The demographic characteristics of each group are presented in Table 1.

The NC group had no memory complaints, scored in the normal range on standardized neuropsychological tests, and had no neurological abnormalities. aMCI patients met the criteria for aMCI initially proposed by Petersen: ${ }^{1} 1$ ) memory complaints, preferably corroborated by an informant; 2) memory impairment relative to age- and education-matched

Table I Mean demographic data and scores (SD) of the MiniMental State Examination of the normal control, amnestic mild cognitive impairment, and Alzheimer's disease groups

\begin{tabular}{llll}
\hline & $\begin{array}{l}\text { NC } \\
(\mathbf{n}=\mathbf{2 0})\end{array}$ & $\begin{array}{l}\text { aMCI } \\
(\mathbf{n}=\mathbf{2 0})\end{array}$ & $\begin{array}{l}\text { AD } \\
(\mathbf{n}=\mathbf{2 0})\end{array}$ \\
\hline Age, years (SD) & $70.8(5.2)$ & $70.7(5.0)$ & $72.4(5.6)$ \\
Female, n (\%) & $\mathrm{II}(55 \%)$ & $9(45 \%)$ & $10(50 \%)$ \\
Years of education (SD) & $1 \mathrm{I} .2(4.7)$ & $11.0(4.2)$ & $7.0(4.2)^{*}$ \\
MMSE scores (SD) & $27.6(2.0)$ & $25.6(2.4)$ & $20.5(3.4)^{*}$ \\
MMSE range (min, max) & 23,30 & 20,29 & 13,24 \\
\hline
\end{tabular}

Note: $* P<0.05$, significantly different from normal controls and amnestic mild cognitive impaired patients in Bonferroni post hoc analysis.

Abbreviations: $A D$, Alzheimer's disease; aMCl, amnestic mild cognitive impairment; NC, normal control; MMSE, Mini-Mental State Examination; min, minimum; max, maximum; SD, standard deviation. 
healthy individuals (below 1.0 standard deviation); 3) intact general cognitive functioning; 4) largely intact activities of daily living; and 5) absence of dementia. AD was diagnosed according to the National Institute of Neurological and Communicative Diseases and Stroke/Alzheimer's Disease and Related Disorders Association criteria for probable AD, ${ }^{35}$ and subjects were mildly demented, scoring 0.5 or 1 on the Clinical Dementia Rating (CDR) global scale. ${ }^{36}$

After 5 years, in eleven of the 20 aMCI patients, AD was newly developed (aMCI converters), seven still had aMCI (aMCI nonconverters), and two were lost to follow-up.

\section{Neuropsychological assessments}

A trained psychologist administered the Korean Mini-Mental State Examination (MMSE), ${ }^{37,38} \mathrm{CDR}$, and neuropsychological memory and visuospatial tests to the subjects. Visuospatial construction and memory were assessed with the simplified Rey figure test (SRFT). ${ }^{39}$ Working memory was examined using the Spatial Span Forward and Backward tasks to validate the VRAM. A trained psychiatrist made the clinical diagnoses in consultation with a consensus conference at which the clinical and neuropsychological data were reviewed.

\section{VRAM task}

An original virtual maze program, which included a maze with six arms and "treasures" at the distal end of three arms, was used as the VRAM test tool (Figure 1). Participants were told that they were in a virtual room with six arms extending from a middle area. The virtual room had various colored objects and visual cues to indicate the relative directions, and the room remained unchanged throughout each trial. Although participants were instructed to find the three treasures as quickly as possible, no time limit was imposed. After discovering all three treasures, the trial ended, and participants returned to the center of the maze to begin the next trial. Five trials were conducted, and the intertrial interval

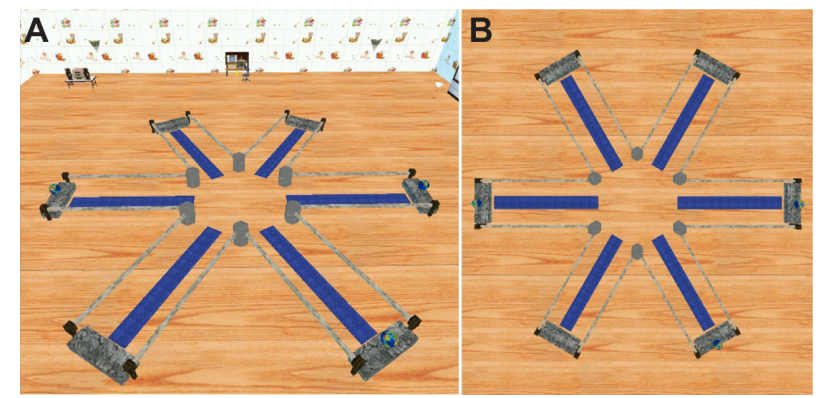

Figure I Virtual radial arm maze test.

Notes: (A) Virtual radial arm maze with six arms in virtual environment. (B) View of virtual radial arm maze from above. was 10 seconds. The same configuration of rewarded arms was used for all participants. This test measured working memory errors by the number of times a subject reentered the same arm; reference memory errors were measured by the number of times a subject reentered the arms with no rewards. ${ }^{22}$ Distance traveled and time required to find all rewards during each trial were also recorded.

\section{Test administration}

All subjects were visually and verbally informed that the test would occur in a virtual room. The apparatus for the VRAM consisted of a desktop computer with a color monitor and a joystick. All participants were instructed to use the joystick to navigate the virtual environment.

All subjects participated in pretrial training before the task. Trained psychologists who did not participate in neuropsychological tests guided the subjects to ensure they were completely familiar with the virtual environment, the rules of the game, and the manipulation of joysticks. Irrespective of disease severity, all subjects were clearly able to perform the tasks without any problems. The total length of training differed across individuals.

Five years after the VRAM test, the aMCI participants were surveyed and categorized into aMCI converters and aMCI nonconverters. The original VRAM trial results previously gathered were compared between the two groups for any significant group effect.

\section{Statistical analysis}

Differences in sex in the three groups were analyzed with chisquare tests. The mean age, years of education, and MMSE scores were compared using analysis of variance (ANOVA) of the three groups. Time latency, distance, number of working memory errors, and number of reference memory errors in the five trials of the VRAM test were compared using repeated measures ANOVA with the Bonferroni adjustment for multiple testing. We used Kendall's tau-b to analyze the correlation between the VRAM results (working and reference memory errors, time, and distance traveled) and other neuropsychological memory tests to examine the concurrent validity of the VRAM. SPSS for Windows, version 16 (SPSS Inc., Chicago, IL, USA) was used for data analyses. $P$-values below 0.05 were considered statistically significant.

\section{Results}

\section{Subjects}

The sample consisted of 60 elderly individuals: 20 with AD, 20 with aMCI, and 20 NC subjects. Demographic 
data and mean MMSE scores are presented in Table 1. The groups had similar mean ages $(F=0.6, P=0.53)$ and sex distributions ( $P=0.81)$, but they differed significantly in years of education $(F=5.8, P<0.05)$ and MMSE scores $(F=38.6, P<0.05)$. Post hoc analysis revealed that those in the AD group were less educated and had lower MMSE scores compared with those in the NC and aMCI groups $(P<0.05)$.

\section{VRAM results}

Repeated measures ANOVAs (Figure 2) revealed a significant main effect of number of trials on working $(F=8.0$, $d f=4$, partial $\left.\eta^{2}=0.37, P<0.05\right)$ and reference $(F=20.0, d f=4$, partial $\left.\eta^{2}=0.60, P<0.05\right)$ memory errors. All three groups committed fewer working and reference memory errors as the trials proceeded. Additionally, we found a significant effect of group on working $\left(F=12.0, d f=2\right.$, partial $\eta^{2}=0.30$, $P<0.05)$ and reference $\left(F=17.7, d f=2\right.$, partial $\eta^{2}=0.38$, $P<0.05)$ memory errors. According to the post hoc analysis, aMCI and NC participants committed a comparable number of working memory errors $(P=0.1)$, but both groups committed fewer working memory errors $(P<0.05)$ than the AD subjects. aMCI subjects committed more reference memory errors than $\mathrm{NC}$ subjects $(P<0.05)$ and committed a similar number of reference memory errors $(P=0.4)$ to AD subjects. We observed a significant main effect of trial on distance traveled to find the rewards $\left(F=20.3, d f=4\right.$, partial $\eta^{2}=0.60$, $P<0.05)$; hence, all three groups traveled shorter distances to find the rewards as the trials progressed. A significant main effect of group on distance traveled to find the rewards ( $F=17.8, d f=2$, partial $\left.\eta^{2}=0.39, P<0.05\right)$ was also observed. Specifically, NC subjects found the rewards after traveling shorter distances than aMCI subjects $(P<0.05)$, and aMCI subjects found the rewards after traveling shorter distances than AD subjects $(P<0.05)$.

Finally, the data reflected a significant main effect of trial on time latency to find the rewards $(F=20.0, d f=4$, partial $\left.\eta^{2}=0.60, P<0.05\right)$. All participants spent less time finding the rewards as the trials proceeded. Moreover, we found a significant group effect of latency $(F=15.8, d f=2$, partial $\left.\eta^{2}=0.36, P<0.05\right)$, revealing that $\mathrm{NC}$ subjects found the rewards more quickly than aMCI subjects $(P<0.05)$, whereas the time to find the rewards did not differ between aMCI and $\mathrm{AD}$ subjects $(P=0.18)$.
A

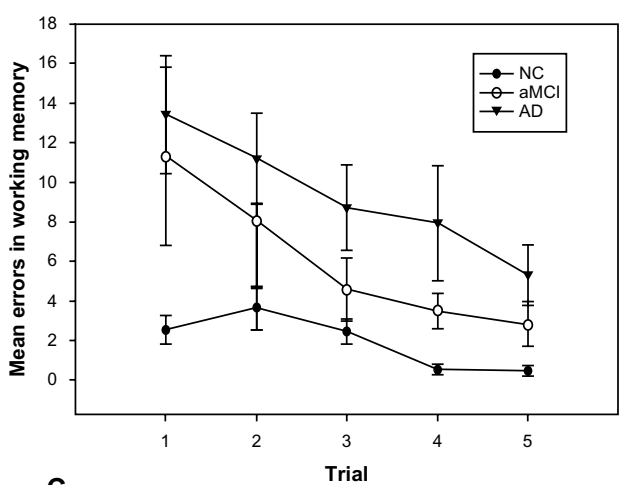

C

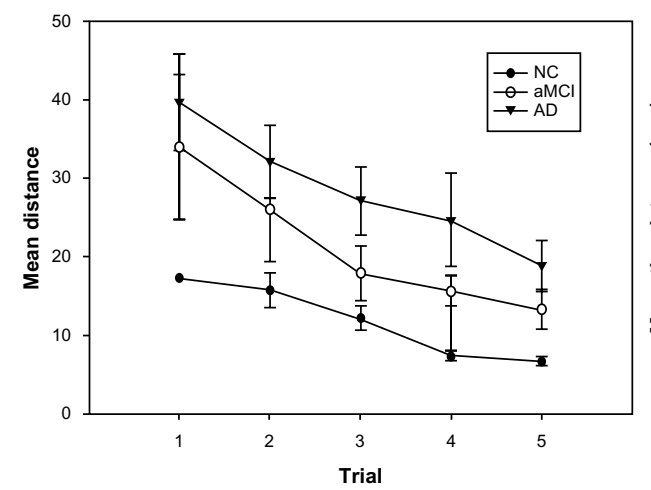

B

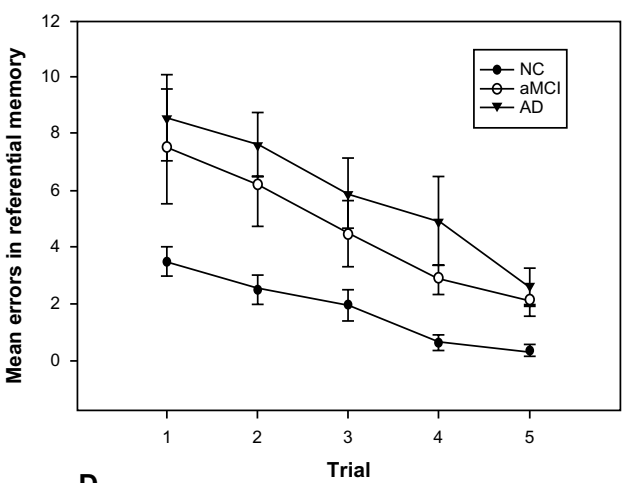

D

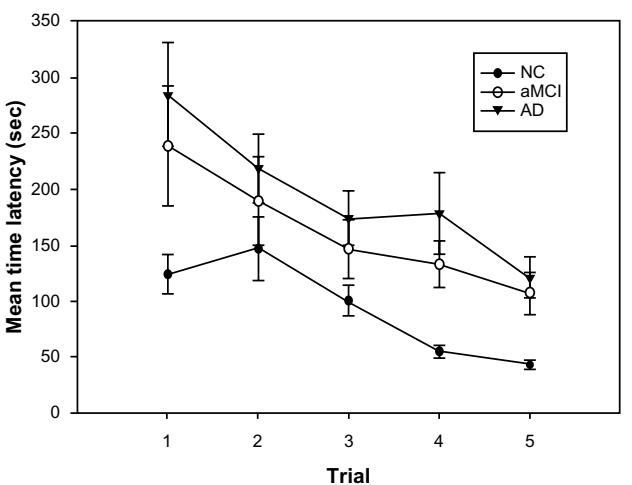

Figure 2 Results of virtual radial arm maze test for the normal control, amnestic mild cognitive impairment, and Alzheimer's disease groups. Notes: (A) Mean working memory errors \pm standard error (SE); (B) mean reference memory errors \pm SE; (C) mean distance \pm SE; and (D) mean time latency \pm SE to find the hidden rewards across trials in the normal control, amnestic mild cognitive impaired, and Alzheimer's disease groups for five trials.

Abbreviations: $\mathrm{AD}$, Alzheimer's disease; aMCl, amnestic mild cognitive impairment; NC, normal control; sec, seconds. 


\section{VRAM results in the aMCl groups}

After following patients who were tested 5 years ago, values of the latency, travel distance, and number of working and reference memory errors were compared between the aMCI converter and aMCI nonconverter groups. Despite the small sample, the aMCI converter group made significantly more reference memory errors on the VRAM than the aMCI nonconverter group (Figure $3, P<0.05$ ). However, the groups did not significantly differ regarding latency $(P=0.08)$, travel distance $(P=0.53)$, and working memory errors $(P=0.1)$.

\section{Average group differences and relationships to other neuropsychological tests}

Table 2 shows the correlations between number of working and reference memory errors in VRAM trials, and neuropsychological test results of Spatial Span Forward and Backward, SRFT copy, SRFT immediate recall, and SRFT delayed recall. The numbers of working and reference memory errors on the VRAM had significant linear correlations with each other and with scores on all neuropsychological tests except the Spatial Span Forward.

A

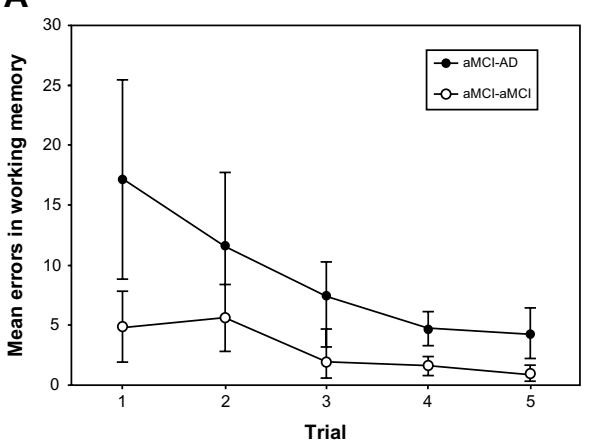

c

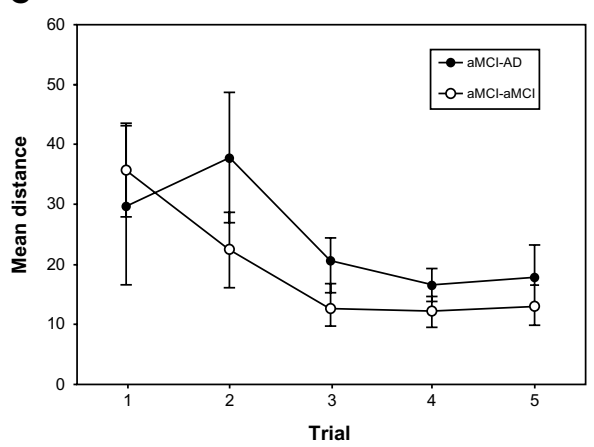

\section{Discussion}

The present study found differences in the spatial memory abilities of NC, aMCI, and AD groups. Participants with aMCI made more spatial reference memory errors than NC subjects, but their spatial working memory seemed intact. AD subjects made more errors in both spatial working and reference memory than did NC subjects. The 5-year follow-up analysis showed that the aMCI converter group made more spatial reference memory errors before developing dementia than the aMCI nonconverter group.

aMCI subjects traveled shorter distances on the VRAM to find the rewards than $\mathrm{AD}$ subjects, and were as slow as AD subjects in finding the rewards. Table 2 presents the results of the VRAM.

Unlike AD, aMCI seems to significantly affect reference memory, but not working memory. Spatial working memory functions have been found to be controlled by the prefrontal cortical area, which is damaged in the progress of $\mathrm{AD},{ }^{32,33}$ and that spatial reference memory functions are affected by hippocampal atrophy that emerges early in the prodromal aMCI stage. ${ }^{28,31}$ A Y-maze study showed that spatial working memory may be preserved longer than spatial reference memory in transgenic mice expressing

\section{B}

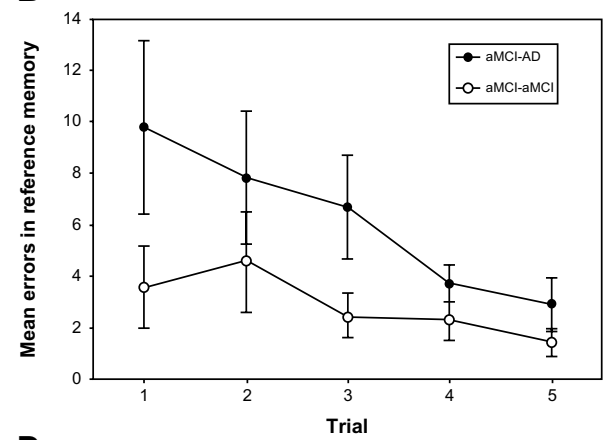

D

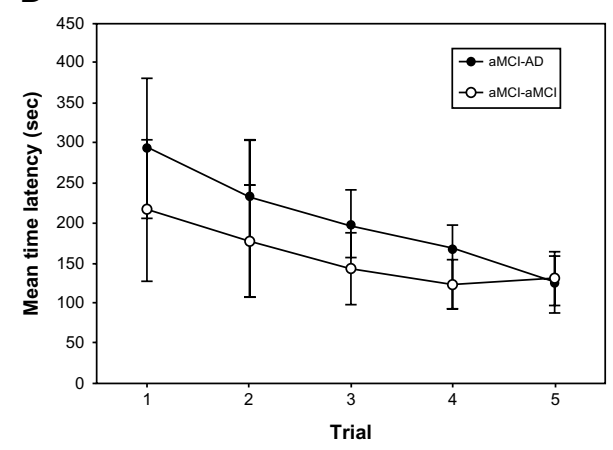

Figure 3 Results of virtual radial arm maze test for the converted and nonconverted amnestic mild cognitive impaired groups.

Notes: (A) Mean working memory errors \pm standard error (SE); (B) mean reference memory errors \pm SE (significant group effect of $P<0.05$ ); (C) mean distance \pm SE; and (D) mean time latency $\pm \mathrm{SE}$ to find the hidden rewards across five trials in converted amnestic mild cognitive impaired (aMCl) and nonconverted aMCl groups within original aMCl group followed-up for 5 years.

Abbreviations: $\mathrm{AD}$, Alzheimer's disease; aMCl, amnestic mild cognitive impairment; sec, seconds. 
Table 2 Correlations between virtual radial arm maze and neuropsychological tests after controlling for years of education

\begin{tabular}{lllll}
\hline & WM errors & RM errors & Time & Distance \\
\hline $\begin{array}{l}\text { Spatial Span } \\
\text { Forward }\end{array}$ & -0.169 & -0.172 & -0.165 & -0.155 \\
$\begin{array}{l}\text { Spatial Span } \\
\text { Backward }\end{array}$ & $-0.286^{\dagger}$ & $-0.334^{\dagger}$ & $-0.25 \mathrm{I}^{\dagger}$ & $-0.307^{\dagger}$ \\
SRFT copy & $-0.356^{\dagger}$ & $-0.3699^{\dagger}$ & $-0.37 \mathrm{I}^{\dagger}$ & $-0.403^{\dagger}$ \\
$\begin{array}{l}\text { SRFT immediate } \\
\text { recall }\end{array}$ & $-0.373^{\dagger}$ & $-0.443^{\dagger}$ & $-0.368^{\dagger}$ & $-0.408^{\dagger}$ \\
SRFT delayed & $-0.389^{\dagger}$ & $-0.45 \mathrm{I}^{\dagger}$ & $-0.418^{\dagger}$ & $-0.410^{\dagger}$ \\
recall & & & & \\
\hline
\end{tabular}

Note: IIndicates that the correlation was significant at the 0.05 level.

Abbreviations: RM, reference memory; SRFT, simplified Rey complex figure test; WM, working memory.

AD pathology. ${ }^{2}$ Therefore, our results suggest that the loss in spatial reference memory may happen earlier during the aMCI stage, before the spatial significant working memory loss associated with the progress of AD appears. In addition, the current results (Figure 2) indicate that spatial reference memory is significantly more impaired in aMCI patients who develop AD within 5 years than in those who do not. VRAM test results may be used to detect aMCI which can progress on to $\mathrm{AD}$.

$\mathrm{aMCI}$ and $\mathrm{AD}$ subjects took significantly longer than $\mathrm{NC}$ subjects to find the rewards. This impairment in locomotion is reportedly caused by a general deterioration in visual attention and visual processing speed, ${ }^{40}$ as well as by deficits in global cognitive ability and perceptual speed. ${ }^{41}$ Patients with aMCI also show attention deficits and process visual stimuli more slowly, although these impairments are less severe in aMCI than in $\mathrm{AD}^{42} \mathrm{AD}$ and aMCI patients are unable to disengage attention and use a visual cue to produce an alerting effect. ${ }^{43}$ Consistent with previous studies, aMCI and AD subjects showed increased time latency, reflecting a decline in visual stimulus processing and attention. Interestingly, aMCI subjects traveled shorter distances, but with a similar latency when compared to AD patients. This may be indicative of intact spatial working memory in aMCI which helped to prevent reentry into previously entered rooms despite decline in the ability to process visually the landmarks in the VRAM.

The results of the VRAM had linear correlations with those of the Spatial Span Backward and SRFT tests. The statistical significance suggests that the VRAM's ability to detect spatial memory deficits may be comparable to that of neuropsychological tests.

However, the spatial working and reference memory errors in the VRAM tests were not significantly correlated with those in the Spatial Span Forward task $(P>0.05)$, which assesses passive short-term memory. Whereas the Spatial Span Backward test involves more active attention processes, ${ }^{44}$ the Spatial Span Forward test assesses only passive attention processes. Previous studies have indicated that passive short-term memories are more resilient and are less likely to be impaired in aMCI and mild $\mathrm{AD},{ }^{31}$ which is why the Spatial Span Forward test cannot examine spatial impairments in $\mathrm{aMCI}$ and $\mathrm{AD}$, and it was not correlated with the VRAM results.

This study has several limitations. Due to the small number of aMCI patients available for follow-up, significant differences in other spatial memory functions between $\mathrm{AD}$ converters and nonconverters may not be observed. In addition, although statistically significant, the linear correlations between VRAM results and the neuropsychological tests (Table 2) were not strong. A larger sample size will improve the quality of statistical data in future investigations. We also assumed that we could completely separate the two different types of spatial memories by counting the number of reentries into emptied arms and selections of wrong arms. However, to test this hypothesis, future research should provide brain imaging analyses to find different brain activation during the occurrence of reference memory and working memory errors.

\section{Conclusion}

aMCI subjects differed from AD subjects in their intact spatial working memory, whereas both aMCI and AD subjects demonstrated impaired spatial reference memory, suggesting that the VRAM can help to distinguish among deficits associated with normal aging, aMCI, and AD. Additionally, the VRAM results regarding reference memory errors may be useful as a clinical marker of possible future development of AD among current aMCI subjects, as shown in the 5-year follow-up. Our study shows that the VRAM can be used to examine impairments in spatial working and reference memories in the early stage of $\mathrm{AD}$.

\section{Acknowledgments}

The English in this document has been checked by at least two professional editors, both native speakers of English (http://textcheck.com). This study was supported by a grant from the Korea Healthcare technology R\&D Project, Ministry for Health and Welfare, Republic of Korea (A070001). The study was conducted at the Seoul National University and Seoul Metropolitan Government - Seoul National University Boramae Medical Center. 


\section{Author contributions}

Dr Jun-Young Lee conceived the study, acquired, and interpreted the data. Ms Sooyeon Kho and Ms Hye Bin Yoo analyzed the data and edited the article. Ms Soowon Park made major revisions to the Introduction section. Dr JungSeok Choi and Jun Soo Kwon acquired the patients and their clinical reports. The corresponding authors Dr Kyung Ryeol Cha and Dr Hee-Yeon Jung designed the research and the experiments. All authors made substantial contributions to conception and design of the paper, acquisition of data, or analysis and interpretation of data, and drafted the article or revised it for critically important content.

\section{Disclosure}

The authors report no conflict of interest in this work. No competing financial interests exist.

\section{References}

1. Petersen RC, Smith GE, Waring SC, Ivnik RJ, Tangalos EG, Kokmen E. Mild cognitive impairment: clinical characterization and outcome. Arch Neurol. 1999;56(3):303-308.

2. Pennanen C, Kivipelto M, Tuomainen S, et al. Hippocampus and entorhinal cortex in mild cognitive impairment and early AD. Neurobiol Aging. 2004;25(3):303-310.

3. Mosconi L, Tsui WH, De Santi S, et al. Reduced hippocampal metabolism in MCI and AD Automated FDG-PET image analysis. Neurology. 2005;64(11):1860-1867.

4. Korf ES, Wahlund LO, Visser PJ, Scheltens P. Medial temporal lobe atrophy on MRI predicts dementia in patients with mild cognitive impairment. Neurology. 2004;63(1):94-100.

5. Maguire EA, Burgess N, Donnett JG, Frackowiak RS, Frith CD, O'Keefe J. Knowing where and getting there: a human navigation network. Science. 1998;280(5365):921-924.

6. Pai MC, Jacobs WJ. Topographical disorientation in communityresiding patients with Alzheimer's disease. Int J Geriatr Psychiatry. 2004;19(3):250-255.

7. Hort J, Laczo J, Vyhnalek M, Bojar M, Bures J, Vlcek K. Spatial navigation deficit in amnestic mild cognitive impairment. Proc Natl Acad Sci U S A. 2007;104(10):4042-4047.

8. Alescio-Lautier B, Michel BF, Herrera C, et al. Visual and visuospatial short-term memory in mild cognitive impairment and Alzheimer disease: role of attention. Neuropsychologia. 2007;45(8):1948-1960.

9. Plancher G, Tirard A, Gyselinck V, Nicolas S, Piolino P. Using virtual reality to characterize episodic memory profiles in amnestic mild cognitive impairment and Alzheimer's disease: Influence of active and passive encoding. Neuropsychologia. 2012;50(5):592-602.

10. Cushman LA, Stein K, Duffy CJ. Detecting navigational deficits in cognitive aging and Alzheimer disease using virtual reality. Neurology. 2008;71(12):888-895.

11. Tippett WJ, Lee JH, Zakzanis KK, Black SE, Mraz R, Graham SJ. Visually navigating a virtual world with real-world impairments: a study of visually and spatially guided performance in individuals with mild cognitive impairments. J Clin Exp Neuropsychol. 2009;31(4): 447-454.

12. Spieker EA, Astur RS, West JT, Griego JA, Rowland LM. Spatial memory deficits in a virtual reality eight-arm radial maze in schizophrenia. Schizophrenia Res. 2012;135(1-3):84-89.

13. Zakzanis KK, Quintin G, Graham SJ, Mraz R. Age and dementia related differences in spatial navigation within an immersive virtual environment. Med Sci Monit. 2009;15(4):CR140-CR150.
14. Bellassen V, Iglói K, de Souza LC, Dubois B, Rondi-Reig L. Temporal order memory assessed during spatiotemporal navigation as a behavioral cognitive marker for differential Alzheimer's disease diagnosis. J Neurosci. 2012;32(6):1942-1952.

15. Moffat SD, Zonderman AB, Resnick SM. Age differences in spatial memory in a virtual environment navigation task. Neurobiol Aging. 2001;22(5):787-796.

16. Astur RS, Taylor LB, Mamelak AN, Philpott L, Sutherland RJ. Humans with hippocampus damage display severe spatial memory impairments in a virtual Morris water task. Behav Brain Res. 2002; 132(1):77-84.

17. Moffat SD, Resnick SM. Effects of age on virtual environment place navigation and allocentric cognitive mapping. Behav Neurosci. 2002;116(5):851-859.

18. Gresack JE, Frick KM. Male mice exhibit better spatial working and reference memory than females in a water-escape radial arm maze task. Brain Res. 2003;982(1):98-107.

19. Levin ED, Kim P, Meray R. Chronic nicotine working and reference memory effects in the 16-arm radial maze: interactions with D1 agonist and antagonist drugs. Psychopharmacology (Berl). 1996;127(1): 25-30.

20. Astur RS, Tropp J, Sava S, Constable RT, Markus EJ. Sex differences and correlations in a virtual Morris water task, a virtual radial arm maze, and mental rotation. Behav Brain Res. 2004;151(1-2): $103-115$.

21. Bimonte HA, Nelson ME, Granholm AC. Age-related deficits as working memory load increases: relationships with growth factors. Neurobiol Aging. 2003;24(1):37-48.

22. Astur RS, St Germain SA, Baker EK, Calhoun V, Pearlson GD, Constable RT. fMRI hippocampal activity during a virtual radial arm maze. Appl Psychophysiol Biofeedback. 2005;30(3):307-317.

23. Becker JT, Walker JA, Olton DS. Neuroanatomical bases of spatial memory. Brain Res. 1980;200(2):307-320.

24. Floresco SB, Seamans JK, Phillips AG. Selective roles for hippocampal, prefrontal cortical, and ventral striatal circuits in radial-arm maze tasks with or without a delay. J Neurosci. 1997;17(5):1880-1890.

25. Hasselmo ME. A model of prefrontal cortical mechanisms for goaldirected behavior. J Cogn Neursci. 2005;17(7):1115-1129.

26. Prior H, Schwegler H, Ducker G. Dissociation of spatial reference memory, spatial working memory, and hippocampal mossy fiber distribution in two rat strains differing in emotionality. Behav Brain Res. 1997;87(2):183-194.

27. Frick KM, Baxter MG, Markowska AL, Olton DS, Price DL. Age-related spatial reference and working memory deficits assessed in the water maze. Neurobiol Aging. 1995;16(2):149-160.

28. Burgess N, Maguire EA, O'Keefe J. The human hippocampus and spatial and episodic memory. Neuron. 2002;35(4):625-641.

29. Courtney SM, Petit L, Maisog JM, Ungerleider LG, Haxby JV. An area specialized for spatial working memory in human frontal cortex. Science. 1998;279(5355):1347-1351.

30. Courtney SM, Ungerleider LG, Keil K, Haxby JV. Transient and sustained activity in a distributed neural system for human working memory. Nature. 1997;386(6625):608-611.

31. Iachini I, Iavarone A, Senese VP, Ruotolo F, Ruggiero G. Visuospatial memory in healthy elderly, AD and MCI: a review. Curr Aging Sci. 2009;2(1):43-59.

32. deIpolyi AR, Rankin KP, Mucke L, Miller BL, Gorno-Tempini ML. Spatial cognition and the human navigation network in AD and MCI. Neurology. 2007;69(10):986-997.

33. Dickerson BC, Bakkour A, Salat DH, et al. The cortical signature of Alzheimer's disease: regionally specific cortical thinning relates to symptom severity in very mild to mild AD dementia and is detectable in asymptomatic amyloid-positive individuals. Cereb Cortex. 2009;19(3): 497-510.

34. Lesher EL, Berryhill JS. Validation of the Geriatric Depression Scale - Short Form among inpatients. J Clin Psychol. 1994;50(2): 256-260. 
35. McKhann G, Drachman D, Folstein M, Katzman R, Price D, Stadlan EM. Clinical diagnosis of Alzheimer's disease: report of the NINCDSADRDA Work Group under the auspices of Department of Health and Human Services Task Force on Alzheimer's Disease. Neurology. 1984;34(7):939-944.

36. Morris JC. The Clinical Dementia Rating (CDR): current version and scoring rules. Neurology. 1993;43(11):2412-2414.

37. Folstein MF, Folstein SE, McHugh PR. "Mini-mental state". A practical method for grading the cognitive state of patients for the clinician. J Psychiatr Res. 1975;12(3):189-198.

38. Lee DY, Lee KW, Lee JH, et al. A normative study of Mini-mental state examination in the Korean elderly. J Korean Neuropsychiatr Assoc. 2002;41(3):508-525.

39. Milberg WP, Hebben N, Kaplan E. The Boston Process Approach to Neuropsychological Assessment. In: Grant I, Adams K, editors. Neuropsychological Assessment of Neuropsychiatric Disorders. New York: Oxford University Press; 1996:58-80.
40. Rizzo M, Anderson SW, Dawson J, Myers R, Ball K. Visual attention impairments in Alzheimer's disease. Neurology. 2000;54(10): 1954-1959.

41. Backman L, Jones S, Berger AK, Laukka EJ, Small BJ. Cognitive impairment in preclinical Alzheimer's disease: a meta-analysis. Neuropsychology. 2005;19(4):520-531.

42. Gorus E, De Raedt R, Lambert M, Lemper JC, Mets T. Reaction times and performance variability in normal aging, mild cognitive impairment, and Alzheimer's disease. J Geriatr Psychiatry Neurol. 2008;21(3): 204-218.

43. Tales A, Snowden RJ, Haworth J, Wilcock G. Abnormal spatial and non-spatial cueing effects in mild cognitive impairment and Alzheimer's disease. Neurocase. 2005;11(1):85-92.

44. Cornoldi C, Mammarella IC. A comparison of backward and forward spatial spans. Q J Exp Psychol (Hove). 2008;61(5):674-682.

\section{Publish your work in this journal}

Neuropsychiatric Disease and Treatment is an international, peerreviewed journal of clinical therapeutics and pharmacology focusing on concise rapid reporting of clinical or pre-clinical studies on a range of neuropsychiatric and neurological disorders. This journal is indexed on PubMed Central, the 'PsycINFO' database and CAS.
The manuscript management system is completely online and includes a very quick and fair peer-review system, which is all easy to use. Visit http://www.dovepress.com/testimonials.php to read real quotes from published authors.

Submit your manuscript here: http://www.dovepress.com/neuropsychiatric-disease-and-treatment-journal 